\title{
Severity of periodontal disease in adult patients with diabetes mellitus in relation to the type of diabetes
}

\author{
Alma Pranckeviciene ${ }^{\mathrm{a}}$, Jolanta Siudikiene ${ }^{\mathrm{a}}$, Rytas Ostrauskas ${ }^{\mathrm{b}}$, Vita Machiulskiene ${ }^{\mathrm{a}}$
}

\begin{abstract}
Background. The purpose of this study was to evaluate associations between diabetes mellitus - related factors and periodontal parameters among adult patients with diabetes mellitus, with respect to type of diabetes.

Methods. Study participants were 179 randomly selected 18-62-year-aged patients with type 1 diabetes mellitus and 87 randomly selected 32-70-year-aged patients with type 2 diabetes. Metabolic control of diabetes was determined by the values of glycosylated haemoglobin ( $\mathrm{HbA} 1 \mathrm{c})$. The periodontal status of all patients was evaluated by simplifying oral debris index (DI-S), probing pocket depth (PPD), gingival recession (GR), clinical attachment level (CAL), and bleeding on probing (BOP). Data analysis was performed with respect to patients' age, diabetes duration, metabolic control level, and diabetes type. Binary regression was used to test relationship of various parameters with CAL.

Results. All periodontal estimates were significantly higher among patients with type 2 diabetes. The periodontal disease was more severe in $>45$-year-aged participants and with DI-S $>1$. In patients with type 1 diabetes, the disease duration $>12$ years was negatively related to most periodontal parameters. No significant correlation between the periodontal estimates and $\mathrm{HbA} 1 \mathrm{c}$ was observed in either group. The significant predictors of severe periodontal disease were type 2 diabetes mellitus ( $\mathrm{OR}=2.356)$, duration of disease $(\mathrm{OR}=1.827)$, high $\mathrm{BOP}(\mathrm{OR}=3.343)$ and $\mathrm{DI}-\mathrm{S}(\mathrm{OR}=2.958)$. Conclusions. Severity of periodontal disease is related to diabetes type, being more pronounced in patients with type 2 diabetes patients than in patients with type 1 diabetes. Dental plaque seems to be the major contributing factor for all patients with progressive periodontitis.
\end{abstract}

Key words: diabetes mellitus type 1, diabetes mellitus type 2, periodontitis

Received: October 22, 2013; Accepted: December 19, 2013

http://dx.doi.org/10.5507/bp.2013.098

${ }^{a}$ Department of Dental and Oral Pathology, Lithuanian University of Health Sciences, Eiveniu 2, 50009-Kaunas, Lithuania

bInstitute of Endocrinology, Lithuanian University of Health Sciences, Eiveniu 2, 50009-Kaunas, Lithuania Corresponding author: Alma Pranckeviciene, e-mail:alma_pranckeviciene@yahoo.com

\section{INTRODUCTION}

Periodontitis and diabetes mellitus (DM) are prevalent chronic diseases that influence patients' health and quality of life. Periodontitis is defined as a chronic bacterial inflammatory process with the symptoms of gingival bleeding, clinical attachment loss, formation of periodontal pockets and gingival recessions leading to destruction of alveolar bone ${ }^{1}$. Although mild forms such as gingivitis or initial loss of periodontal attachment are very common in all age cohorts, advanced stages of periodontitis usually affect a relatively small proportion of the population ${ }^{1,2}$. It seems that there is no direct correlation between chronic gingival inflammation and further progression of periodontal breakdown, and lack of evidence on prognostic indicators of severe periodontal disease in those people who develop rapidly progressing periodontal symptoms (2). $\mathrm{DM}$ is a metabolic disorder characterized by hyperglycaemia, leading to impaired metabolism of glucose, lipids and proteins ${ }^{3}$. In 2011 DM affected 6\% of the European population from 20 to 79 years, meaning 52.6 million people with $\mathrm{DM}^{4}$. According to the International Diabetes Federation this number may rise to $7.1 \%$, or 64 million by 2030 (ref. ${ }^{4}$ ). Two types of DM differ with respect to physiopathology: type 1 DM (T1DM), an autoimmune disorder that leads to destruction of pancreatic $\beta$ cells and thus total loss of insulin secretion; and type 2 DM (T2DM) that is linked to insulin resistance ${ }^{5}$. Chronic hyperglycaemia leads to long-term micro and macro vascular complications such as retinopathy, nephropathy, neuropathy and cardiovascular diseases, resulting in increased morbidity and premature mortality ${ }^{6}$. A large number of epidemiological studies have demonstrated that periodontitis is more prevalent and severe in patients with DM when compared to persons without DM ( ref. $^{7}$ ). Moreover, $\mathrm{DM}$ has been suggested as a significant risk factor for periodontal disease ${ }^{8}$. The mechanism of DM effect on the course of periodontal pathology is still not clear, but the severity of both diseases (DM and periodontitis) was shown to be dependent on various risk factors such as duration and metabolic control of DM as well as the patient's age, social behaviour, oral hygiene level and certain aggravating factors (e.g., smoking) (ref. ${ }^{6,7}$ ). The majority of research has focused on the relationship between periodontitis and DM of one particular type (either T1DM or T2DM) while the possible differences in the course of periodontal disease with respect to the different physiopathology of DM have not been studied. The aim of the 
study was to evaluate associations between DM - related factors and periodontal parameters among adult patients with DM, with respect to type of diabetes.

\section{MATERIALS AND METHODS}

\section{Study population}

The study participants were adult patients with diabetes mellitus, within the age range of $18-70$ years, and residing in the second largest city of Lithuania, Kaunas. All study participants were diagnosed with diabetes mellitus for at least, one year. Edentulous patients, pregnant and lactating women were excluded from the study.

Depending on the type of DM (T1DM and T2DM), two study groups were formed. The study sample calculation for patients with T1DM was based on the $60 \%$ prevalence of periodontitis in DM patients estimated in previous studies, with the margin of error $5 \%$ and confidence level 95\% (ref. $^{9}$ ). Only 87 patients with T2DM agreed to participate in the study. However, when the outcome parameters as clinical attachment level and periodontal pocket depth were evaluated and compared between the two study groups, with the $95 \%$ confidence interval and margin of error $5 \%$, the study power was equal to 1.0, thus considered sufficient. The personal information such as date of birth, diagnosis of DM, the duration as well as deleterious habits, were retriever either from the Register database or from the medical records.

\section{Ethical considerations}

The informed consent of all subjects was obtained, and the study protocol was approved by the Ethical Committee of Biomedical Studies of Kaunas region, Lithuania (protocol Nr BE-2-14).

\section{Clinical examinations}

The level of metabolic control of DM of the study participants was determined by measuring the glycosylated haemoglobin (HbA1c) (ref.5). The blood samples were collected by finger stick and analysed using a immunochemical technique with the analyser DCA-2000, Bayer Diagnostics, Germany.

Periodontal examinations of all participants were performed under standardized conditions (a regular dental chair equipped with a light source and air spray), by the same examiner periodontologist (AP).

The following periodontal parameters were recorded using a dental mirror and a ball-pointed calibrated periodontal probe "Hu-Friedy" measured every millimeter:

1. The presence of dental plaque was determined using the simplified oral debris index (DI-S), as described by Greene and Vermillion ${ }^{10}$. The individual DI-S was calculated by adding the scores for six different tooth surfaces and dividing by the number of teeth examined. The nominal scale for evaluation of DI-S was: excellent - 0; good - 0.1-0.6; fair - 0.7-1.8; poor - 1.9-3.0.

2. Probing pocket depth (PPD). PPD was measured on every tooth (excluding the remaining roots), on six points ( 3 buccal and 3 lingual or palatal) from the gingival margin to the base of the pocket or crevice.

3. Gingival recession (GR) was measured from the cement-enamel junction to the margin of the gingiva (on the six points of every tooth).

4. Clinical attachment level (CAL). CAL was calculated as a mathematical sum of PPD and GR.

5. Bleeding on probing (BOP) was estimated as a dot of blood at the base of the pocket or crevice after 30 seconds of gentle probing with the periodontal probe and calculated as a percentage of all bleeding sites.

A calibration session was performed prior to the study for the clinical examiner (AP), by examining 30 subjects twice, with an interval of 72 h. Cohen's kappa for presence/absence of plaque, gingival bleeding, probing depth, clinical attachment loss and gingival recession was calculated. The resulting kappa values ranged between 0.87 and 0.93 showing a good intra-examiner agreement.

\section{Statistical analysis}

The data were analysed by means of Statistical Package for Social Sciences program (SPSS 19.0, Chicago III, USA). For the analysis, the patients were grouped by age as follows: $<45$ years and $>45$ years, and duration of DM was split into groups $<12$ years, and $>12$ years, $\mathrm{HbA} 1 \mathrm{c}$ values were dichotomized to $<7 \%$ (good/fair metabolic control) and $>7 \%$ (poor metabolic control). For estimation of the periodontal parameters, DI-S values were defined as $<1$ and $>1$ ( $>1$ indicating poor oral hygiene), the GR values $>1 \mathrm{~mm}$ were assumed to indicate presence of gingival recession; the CAL values above $5 \mathrm{~mm}$ were assumed to indicate severe periodontal destruction. The threshold for PPD values was defined at $5 \mathrm{~mm}$, assuming that the values greater than $5 \mathrm{~mm}$ indicated severe periodontal destruction, and the threshold for BOP values was defined as $50 \%$ assuming that the values greater than $50 \%$ indicated severe gingivitis.

The mean values and standard deviations of the clinical parameters were calculated. The statistical significance of difference in proportion was tested by the Chi-square test. Continuous data were tested for normality with the Kolmogorov-Smirnov test. Statistical testing between the groups was performed using Student's $t$ test. For the data that were not normally distributed, Mann-Whitney $U$ test was applied. Binary regression analysis was used to test relationship of various parameters with CAL. The probability of $>20$ sites with CAL greater than $5 \mathrm{~mm}$ was calculated using logistic regression analysis, including odds ratio (OR) and confidence interval (95\% CI). For complex evaluation of probability, the multivariate logistic regression model was used. Two-sided p-values less than 0.05 were considered as statistically significant. 


\section{RESULTS}

Patients with T2DM were significantly older than patients with T1DM.

The mean duration of the DM was significantly loněger and the mean $\mathrm{HbAlc}$ values were higher in T1DM group, as compared to the T2DM group. Smoking was significantly more frequent among patients with T1DM than among patients with T2DM (Table 1).

As shown in Table 2, the mean and relative values of all the estimated periodontal parameters were significantly higher in T2DM group-indicating more severe periodontal pathology among patients with T2DM.

Tables 3 and 4 demonstrate that the estimated periodontal parameters were related to the patients' age and oral hygiene level in both T1DM and T2DM groups, for those older than 45 years and with DI-S $>1$ the periodontal disease was more severe. In T1DM group, the DM duration > 12 years had a negative impact on most of the estimated periodontal parameters as well. No significant correlation between the periodontal estimates and the HbA1c levels was observed in both groups of DM subjects.

Moreover, the T2DM smokers had significantly lower mean BOP and CAL values than non-smokers.

As shown in Table 5, the significant predictors of severe periodontal disease were the type of DM, duration of DM as well as bleeding on probing and oral hygiene level expressed by DI-S index.

Thus, for a T2DM patient, the risk of having severe periodontitis was two times higher than for T1DM.

Long duration of DM had almost a twofold risk, while the periodontal parameters DI-S $>1$ and extensive BOP $(>50 \%$ ) had a twofold risk to develop severe periodontal disease. However, the increased HbAlc above 7\% did not seem to influence the course of periodontitis.

\section{DISCUSSION}

The interrelationship of DM and periodontal disease is complex and, despite being extensively investigated, difficult to explain conclusively. As pointed out by Mealey and Oates, 2006, evaluation of the accumulated research should be done with caution as considerable diversity of populations, varying definitions of the parameters under investigation; small numbers of study subjects may play a role in interpretation of the results ${ }^{9}$. The mechanisms by which DM influences the periodontium are stated to be similar to the pathogenesis of the micro- and macro vascular complications of DM such as cardiopathy, nephropathy, and retinopathy ${ }^{3}$. DM negatively affects neutrophil adherence, chemotaxis, and phagocytosis which facilitates bacterial persistence and increases periodontal destruction $^{11}$. On the other hand, monocytes and macrophages become hyper-responsive and produce increased amounts of pro-inflammatory cytokines and mediators which appear also in gingival crevice ${ }^{12}$. Formation of advanced glycosylation end-products are a critical link in many complications of DM, and their deleterious effects also appear in periodontal tissues ${ }^{13}$.

We examined patients with both types of DM, and analysed possible associations of the periodontal parameters with the type of DM. The results have shown the periodontal disease to be significantly more severe in patients with T2DM with respect to all periodontal parameters estimated (DI-S, BOP, PPD, CAL, GR and tooth loss). These findings are in agreement with the data

Table 1. Description of the study groups according to the parameters of diabetes mellitus and smoking habit.

\begin{tabular}{llccc}
\hline & & T1DM group & T2DM group & $P$ \\
\hline Age, years & Mean \pm SD & $38.4 \pm 11.3$ & $55.1 \pm 6.2$ & $<0.001$ \\
Duration of diabetes (years) & Mean \pm SD & $16.4 \pm 10.9$ & $9.4 \pm 6.2$ & $<0.001$ \\
HbA1c level, \% & Mean \pm SD & $8.3 \pm 1.6$ & $7.2 \pm 1.2$ & $<0.001$ \\
Smoking & n (\%) & $58(32.4)$ & $11(12.6)$ & $<0.001$ \\
\hline
\end{tabular}

T1DM - type 1 diabetes mellitus; T2DM - type 2 diabetes mellitus; SD - standard deviation.

Table 2. Description of periodontal parameters in the study groups.

\begin{tabular}{lllll}
\hline & & T1DM group & T2DM group & $P$ \\
\hline DI-S & Mean \pm SD & $1.1 \pm 0.6$ & $1.2 \pm(0.7)^{*}$ & $<0.05$ \\
BOP $\%$ & Mean \pm SD & $46.7 \pm 29.3$ & $54.5 \pm 29.3$ & $<0.05$ \\
PPD $>5 \mathrm{~mm}$ & $\mathrm{n}(\%)$ & $127(70.9)$ & $77(88.5)$ & $<0.001$ \\
$\mathrm{CAL}>5 \mathrm{~mm}$ & $\mathrm{n}(\%)$ & $137(76.5)$ & $83(95.4)$ & $<0.001$ \\
GR & $\mathrm{n}(\%)$ & $134(74.9)$ & $79(90.8)$ & $<0.001$ \\
No of missing teeth & Mean \pm SD & $7.5 \pm 6.4$ & $11.9 \pm 5.9$ & $<0.001$ \\
\hline
\end{tabular}

T1DM - type 1 diabetes mellitus; T2DM - type 2 diabetes mellitus; DI-S -Debris index; BOP - Bleeding on probing; PPD - Probing pocket depth; CAL - Clinical attachment level; GR - Gingival recession. 
supported by the meta-analysis of earlier studies ${ }^{14}$. The first explanation at hand for these findings could be age of patients as T2DM patients were significantly older than the T1DM subjects. However, the patients with T1DM were observed to have longer duration of DM, and higher mean values of $\mathrm{HbAlc}$ which contradicts the commonly reported message about these factors increasing risk for periodontal disease.

Age of the patients did appear a significant parameter in our study, when both groups of patients (T1DM and T2DM) had been examined. Those above 45 years of age had generally lower values of the periodontal parameters, irrespective of what type of DM they had been diagnosed with. When age was entered into the binary logistic regression model, it appeared to be a significant risk factor for development of severe periodontal disease.

Previous studies of patients with DM have demonstrated a negative correlation of the periodontal state with age. They estimated greater bone loss in patients above the age of 55 years and found the older age to be significantly associated with severity of periodontal disease $^{15-17}$. Some studies indicated that patient's aging may have more impact on the development of periodontitis than the state of DM (ref. ${ }^{18}$ ). In contrast, Abdellatif and

Table 3. Relation of the periodontal parameters with type 1 diabetes mellitus patients`age, duration of diabetes, metabolic control, smoking and oral hygiene.

\begin{tabular}{|c|c|c|c|c|c|}
\hline & $\begin{array}{c}\mathrm{PPD}>5 \mathrm{~mm} \\
\mathrm{n}(\%)\end{array}$ & $\begin{array}{c}\mathrm{CAL}>5 \mathrm{~mm} \\
\mathrm{n}(\%)\end{array}$ & $\begin{array}{c}\text { BOP } \\
\text { Mean } \pm \text { SD }\end{array}$ & $\begin{array}{c}\text { GR } \\
\mathrm{n}(\%)\end{array}$ & $\begin{array}{l}\text { Tooth loss } \\
\text { Mean } \pm \text { SD }\end{array}$ \\
\hline \multicolumn{6}{|c|}{ Patient's age } \\
\hline$>45$ years & $47(87.0)$ & $50(92.6)$ & $58.9 \pm 30.9$ & $51(94.4)$ & $12.1 \pm 7.8$ \\
\hline$<45$ years & $80(64.0)^{* *}$ & $87(69.6)^{* *}$ & $41.4 \pm 26.9 * *$ & $83(66.4)^{* *}$ & $5.6 \pm 4.6 * *$ \\
\hline \multicolumn{6}{|c|}{ Duration of diabetes } \\
\hline$>12$ years & $81(78.6)$ & $85(82.5)$ & $47.4 \pm 30.2$ & $82(79.6)$ & $8.7(6.6)$ \\
\hline$<12$ years & $46(60.5)^{* *}$ & $52(68.4)^{*}$ & $45.7 \pm 28.1$ & $52(68.4)$ & $5.9 \pm 5.9 * *$ \\
\hline \multicolumn{6}{|c|}{ HbA1c level } \\
\hline$>7 \%$ & $11(67.6)$ & $114(78.6)$ & $47.5 \pm 28.7$ & $111(76.6)$ & $7.5 \pm 5.9$ \\
\hline$<7 \%$ & $104(71.7)$ & $23(67.6)$ & $43.4 \pm 31.7$ & $23(67.6)$ & $7.8 \pm 8.2$ \\
\hline \multicolumn{6}{|l|}{ Smoking } \\
\hline Yes & $39(67.2)$ & $43(74.1)$ & $47.4 \pm 30.1$ & $50(72.5)$ & $7.6 \pm 6.9$ \\
\hline No & $88(72.7)$ & $94(77.7)$ & $46.3 \pm 28.9$ & $163(82.7)$ & $7.5 \pm 6.3$ \\
\hline \multicolumn{6}{|l|}{ DI-S } \\
\hline$>1$ & $70(87.5)$ & $74(92.5)$ & $62.3 \pm 27.7$ & $14(82.5)$ & $9.5 \pm 6.9$ \\
\hline$<1$ & $57(57.6)^{* *}$ & $63(63.6)^{* *}$ & $34.1 \pm 23.9 * *$ & $68(68.7)^{*}$ & $5.9 \pm 6.9 *$ \\
\hline
\end{tabular}

Statistically significant difference between the groups, with respect to age, DM duration, HbA1c level, smoking habit and DI-S, * P<0.05; ** P<0.001. DI-S -Debris index; BOP - Bleeding on probing; PPD - Probing pocket depth; CAL - Clinical attachment level; GR - Gingival recession.

Table 4. Relation of the periodontal parameters with type 2 diabetes mellitus patients' age, duration of diabetes, metabolic control, smoking and oral hygiene.

\begin{tabular}{|c|c|c|c|c|c|}
\hline & $\begin{array}{c}\text { PPD }>5 \mathrm{~mm} \\
\mathrm{n}(\%)\end{array}$ & $\begin{array}{c}\text { CAL }>5 \mathrm{~mm} \\
\mathrm{n}(\%)\end{array}$ & $\begin{array}{c}\text { BOP } \\
\text { Mean } \pm \text { SD }\end{array}$ & $\begin{array}{c}\text { GR } \\
\text { n (\%) }\end{array}$ & $\begin{array}{l}\text { Tooth loss } \\
\text { Mean } \pm \text { SD }\end{array}$ \\
\hline \multicolumn{6}{|c|}{ Patient's age } \\
\hline$>45$ years & $70(89.7)$ & $76(97.4)$ & $56.8 \pm 29.0$ & $73(93.6)$ & $12.2 \pm 5.9$ \\
\hline$<45$ years & $7(77.8)$ & $7(77.8 \%)^{* *}$ & $34.0 \pm 24.5^{*}$ & $6(66.7)^{* *}$ & $9.4 \pm 4.2$ \\
\hline \multicolumn{6}{|c|}{ Duration of diabetes } \\
\hline$>12$ years & $18(90.0)$ & $19(95.0)$ & $56.4 \pm 29.5$ & $19(95.0)$ & $11.6 \pm 5.5$ \\
\hline$<12$ years & $59(88.1)$ & $64(95.5)$ & $53.9 \pm 29.4$ & $60(89.6)$ & $11.9 \pm 6.0$ \\
\hline \multicolumn{6}{|c|}{ HbA1c level } \\
\hline$>7 \%$ & $33(86.8)$ & $36(94.7)$ & $58.3 \pm 30.5$ & $34(89.5)$ & $12.6 \pm 5.5$ \\
\hline$<7 \%$ & $44(89.8)$ & $47(95.9)$ & $51.5 \pm 28.3$ & $45(91.8)$ & $11.3 \pm 6.1$ \\
\hline \multicolumn{6}{|l|}{ Smoking } \\
\hline Yes & $8(72.7)$ & $8(72.1)$ & $29.9 \pm 20.8$ & $10(90.9)$ & $10.8 \pm 3.4$ \\
\hline No & $69(90.8)$ & $75(98.7)^{* *}$ & $58.0 \pm 8.7 * *$ & $69(90.8)$ & $12.1 \pm 6.1$ \\
\hline \multicolumn{6}{|l|}{ DI-S } \\
\hline$>1$ & $46(95.8)$ & $48(100)$ & $69.0 \pm 24.9$ & $43(89.6)$ & $12.8 \pm 5.9$ \\
\hline$<1$ & $31(79.5)^{*}$ & $35(89.7)^{*}$ & $36.6 \pm 23.9 * *$ & $36(92.3)$ & $10.8 \pm 5.7$ \\
\hline
\end{tabular}

Statistically significant difference between the groups, with respect to age, DM duration, HbA1c level, smoking habit and DI-S, * P<0.05; ** P<0.001. DI-S -Debris index; BOP - Bleeding on probing; PPD - Probing pocket depth; CAL - Clinical attachment level; GR - Gingival recession. 
Burt, 1987, concluded that age was much less of a factor determining periodontal disease when oral hygiene status had been considered ${ }^{19}$. Some authors suggest another potential explanation for the increased severity of periodontal disease among patients with T2DM: this could be a low-grade chronic inflammatory state caused by obesity common among patients with T2DM (ref.,6). It has been reported that subjects with hyperlipidaemia suffer significantly more severe periodontitis; moreover, periodontal destruction positively correlates to plasma lipid levels ${ }^{20}$. Adipocytes have a metabolic activity and produce proinflammatory cytokines such as TNF- $\alpha$ and IL-6 (ref. ${ }^{21}$ ). Effective management of hyperglycaemia allows ameliorating the serum lipid disbalance for T1DM subjects, but for T2DM patient's hyperlipidaemia still persists despite glycemic control ${ }^{22}$.

Considering the importance of the duration of DM for the development of periodontal pathology in DM subjects, findings of various studies are controversial. Some studies indicate that the time since diagnosis of DM was the most significant factor associated with periodontal parameters such as PPD, GR and CAL (ref. ${ }^{15}$ ). These data receive support from some researchers ${ }^{23}$, while others observed no significant relationship between duration of DM and the periodontal state ${ }^{24}$. In one study tooth loss was reported to be associated with extensive periodontal disease as well as with prolonged duration of DM (ref. ${ }^{16}$ ). In the present study, the duration of DM appeared to be a predictive factor for the development of severe periodontitis. However, the significance of this prediction was marginal, even though it had a significant correlation with high PPD, CAL values and with a mean number of lost teeth in patients with T1DM (Table 3). On the other hand, among patients with T2DM the impact of duration of DM on the periodontal parameters was not significant, perhaps due to the fact that the mean duration of disease was significantly shorter than among patients with T1DM, and less than 10 years.

Metabolic control as determined by the HbAlc value was found to have no significant influence on the periodontal state either among T1DM or among patients with T2DM in our study. However, in T1DM patients HbA1c values $>7 \%$ tended to have increased CAL and BOP values, while in the T2DM group the same HbA1c values were linked to the increased BOP and tooth loss.

The degree of glycemic control is claimed to be an important variable in the relationship between DM and periodontal diseases, with more severe conditions being seen in those patients with poor control ${ }^{25,26}$. However, this is not a general rule. The data from several studies contradict this statement ${ }^{27,28}$. According to other study one, people with an increased mean PPD had significantly higher $\mathrm{HbAlc}$ values. However, no correlation of HbAlc levels with mean CAL, GR or BOP values was estimated ${ }^{29}$. In another study, HbA1c values were significantly associated with CAL, but not with other periodontal parameters ${ }^{24}$.

The weakness of the present study is that we obtained the HbA1c values only for the past 3 months. According to studies in which the impact of metabolic control was proven, high blood glucose sustained over time gives rise to chronic inflammatory mediator secretion and therefore to increased periodontal response. Moreover, there is individual patient variability in the degree to which glycemic control influences periodontal state, therefore, patients with well-controlled DM can have periodontal diseases just as those with poorly controlled DM can have a healthy periodontium ${ }^{30}$.

Researchers find smoking to be a risk factor for periodontal disease; smokers suffer more severe periodontitis and show more resistance to periodontal therapy ${ }^{31}$.

In our study, the effect of smoking on periodontal destruction did not reach statistical significance in the T1DM group; however, in T2DM group smokers had a significantly lower mean BOP and percentage of CAL >5 $\mathrm{mm}$. Lower levels of gingival bleeding could be explained by the vasoconstrictive effect of nicotine ${ }^{32}$. On the other hand, there were only 11 smokers $(12.6 \%)$ among patients with T2DM in the present study and, therefore, a small number of the subjects may produce ambiguous results.

The role of microbial plaque in the aetiology of periodontal disease is undisputed. An overhelming evidence about the close relationship between dental plaque and gingivitis has been accumulated since 1965 when the first report was published ${ }^{33}$. However, a much less pronounced relationship between dental plaque and severe periodontitis has been demonstrated. Moreover, it has been stated that dental plaque by itself is a poor predictor of subsequent periodontitis disease activity, with many additional risk factors playing role in the pathogenesis ${ }^{34}$. In the present study, high values of the simplified oral debris index (DI-S) have been estimated to have the greatest risk to develop severe periodontal disease. The increased DI-S values were associated with all periodontal parameters measured in patients with T1DM as well as in

Table 5. Logistic regression models for predictors of severe periodontal disease ( $\geq 20$ sites with clinical attachment $(\mathrm{CAL})$ level $>5 \mathrm{~mm}$ ).

\begin{tabular}{lccc}
\hline Covariate & Odds ratio & $95 \%$ CI & $P$ \\
\hline T2DM & 2.356 & $1.208-4.594$ & 0.01 \\
BOP $(>50 \%)$ & 3.343 & $1.832-6.100$ & $<0.001$ \\
HbA1c $(>7 \%)$ & 0.658 & $0.363-1.190$ & 0.166 \\
Duration of the disease $(>12$ yrs $)$ & 1.827 & $1.009-3.310$ & 0.047 \\
DI-S $(>1)$ & 2.958 & $1.625-5.382$ & 0.001 \\
\hline
\end{tabular}

T2DM - type 2 diabetes mellitus; BOP - Bleeding on probing; DI-S -Debris index. 
patients with T2DM, except for the formation of gingival recessions in the latter group. Bleeding on probing for all patients with DM examined, was a significant predictor of severe periodontal destruction as well. However, bleeding on probing first of all, reflects gingival inflammation which is a common response reaction to microbial plaque accumulation ${ }^{35}$. Therefore, even considering the multifactorial nature of periodontal disease and possibly systemic mechanisms of DM involvement in pathogenesis of periodontitis, the major role of oral hygiene seems to be the key factor in progression of severe periodontal destruction. As pointed out by Mealey, 2006, systemic conditions play more modifying rather than a primary causative role ${ }^{30}$.

\section{CONCLUSIONS}

The results demonstrate that severity of periodontal disease is more pronounced in patients with type 2 diabetes mellitus. However, along with potential systemic or time-related factors such as patient age and disease duration, the dental plaque level seems to be the major contributing factor for all patients with progressing periodontal disease.

\section{ACKNOWLEDGEMENTS}

The study was supported by the Research Foundation of Lithuanian University of Health Sciences.

\section{CONFLICT OF INTEREST STATEMENT}

The authors stated that there are no conflicts of interest regarding the publication of this article.

\section{REFERENCES}

1. Bascones-Martinez A, Matesanz-Perez P, Escribano-Bermejo M González-Moles MÁ, Bascones-Ilundain J, Meurman JH. Periodontal disease and diabetes-Review of the Literature. Med Oral Patol Oral Cir Bucal 2011;16(6):e722-9.

2. Sheiham A, Netuveli GS. Periodontal disease in Europe. Periodontology 2000 2002;29:104-21.

3. Mealey BL, Ocampo GL. Diabetes mellitus and periodontal disease. Periodontology 2000 2007; 44:127-53.

4. Whiting DR, Guariguata L, Weil C, Shaw J. IDF diabetes atlas: global estimates of the prevalence of diabetes for 2011 and 2030. Diabetes Res Clin Pract 2011;94(3):311-21.

5. American Diabetes Association. Standards of medical care in diabetes-2007. Diabetes Care 2007;30(Suppl 1):S4-S41.

6. Lalla E, Papapanou PN. Diabetes mellitus and periodontitis: a tale of two common interrelated diseases. Nat Rev Endocrinol 2011;7(12):738-48.

7. Khader YS, Dauod AS, El-Qaderi SS, Alkafajei A, Batayha WQ. Periodontal status of diabetics compared with nondiabetics: a metaanalysis. J Diabetes Complications 2006;20(1):59-68.

8. Löe H. Periodontal disease. The sixth complication of diabetes mellitus. Diabetes Care 1993;16(1):329-34.

9. Mealey B, Oates TW. Diabetes mellitus and periodontal disease. J Periodontol 2006; 77(8):1289-303.
10. Greene JC, Vermillion JR. The simplified oral hygiene index. J Am Dent Assoc 1964:68:7-13.

11. Theule-Grant DA. Periodontal disease, diabetes, and immune response: a review of current concepts. J West Soc Periodontol Periodontal Abstr 1996;44(3):69-77.

12. Salvi GE, Collins JG, Yalda B, Arnold RR, Lang NP, Offenbacher S. Monocytic TNF alpha secretion patterns in IDDM patients with periodontal diseases. J Clin Periodontol 1997;24(1):8-16.

13. Schmidt AM, Weidman E, Lalla E, Yan SD, Hori O, Cao R, Brett JG, Lamster IB. Advanced glycation endproducts (AGEs) induce oxidant stress in the gingiva: a potential mechanism underlying accelerated periodontal disease associated with diabetes. J Periodontal Res 1996;31(7):508-15.

14. Chávarry NG, Vettore MV, Sansone C, Sheiham A. The relationship between diabetes mellitus and destructive periodontal disease: a meta-analysis. Oral Health Prev Dent 2009;7(2):107-27.

15. Cerda J, Vázquez de la Torre C, Malacara JM, Nava LE. Periodontal disease in non-insulin dependent diabetes mellitus (NIDDM). The effect of age and time since diagnosis. J Periodontol 1994;65(11):991-5.

16. Moore PA, Weyant RJ, Mongelluzzo MB, Myers DE, Rossie K, Guggenheimer J, Block HM, Huber H, Orchard T. Type 1 diabetes mellitus and oral health: assessment of periodontal disease. J Periodontol 1999;70(4):409-17.

17. Choi YH, McKeown RE, Mayer-Davis EJ, Liese AD, Song KB, Merchant AT. Association between periodontitis and impaired fasting glucose and diabetes. Diabetes Care 2011;34(2):381-6.

18. Yalda B, Offenbacher $S$, Collins $G$. Diabetes as a modifier of periodontal disease expression. Periodontol 2000 1994;6:37-49.

19. Abdellatif HM, Burt BA. An epidemiological investigation into the relative importance of age and oral hygiene status as determinants of periodontitis. J Dent Res 1987;66(1):13-8.

20. Penumarthy S, Penmetsa GS, Mannem S. Assessment of serum levels of triglycerides, total cholesterol, high-density lipoprotein cholesterol, and low-density lipoprotein cholesterol in periodontitis patients. J Indian Soc Periodontol 2013;17(1):30-5.

21. Festa A, D'Agostino RJr, Howard G, Mykkänen L, Tracy RP, Haffner SM. Chronic subclinical inflammation as part of the insulin resistance syndrome: the Insulin Resistance Atherosclerosis Study (IRAS). Circulation 2000;102(1):42-7.

22. Howard BV, Schneiderman N, Falkner B, Haffner SM, Laws A. Insulin, health behaviors, and lipid metabolism. Metabolism 1993;42(Suppl 1):25-35.

23. Silvestre FJ, Miralles L, Llambes F, Bautista D, Solá-Izquierdo E, Hernández-Mijares A. Type 1 diabetes mellitus and periodontal disease: relationship to different clinical variables. Med Oral Patol Oral Cir Bucal 2009;14(4):E175-9.

24. Bridges RB, Anderson JW, Saxe SR, Gregory K, Bridges SR. Periodontal status of diabetic and non-diabetic men: effects of smoking, glycemic control, and socioeconomic factors. J Periodontol 1996;67(11):1185-92.

25. Dakovic D, Pavlovic MD. Periodontal disease in children and adolescents with type 1 diabetes in Serbia. J Periodontol 2008; 79(6):98792.

26. Campus G, Salem A, Uzzau S, Baldoni E, Tonolo G. Diabetes and periodontal disease: a case-control study. J Periodontol 2005;76(3):41825.

27. Noack B, Jachmann I, Roscher S, Sieber L, Kopprasch S, Lück C, Hanefeld M, Hoffmann T. Metabolic diseases and their possible link to risk indicators of periodontitis. J Periodontol 2000;71(6):898-903.

28. Saito T, Shimazaki Y, Kiyohara Y, Kato I, Kubo M, lida M, Yamashita Y. Relationship between obesity, glucose tolerance, and periodontal disease in Japanese women: the Hisayama study. J Periodontal Res 2005;40(4):346-53.

29. Chen L, Wei B, Li J, Liu F, Xuan D, Xie B, Zhang J. Association of periodontal parameters with metabolic level and systemic inflammatory markers in patients with type 2 diabetes. J Periodontol 2010;81(3):364-71.

30. Mealey BL. Periodontal disease and diabetes. A two-way street. J Am Dent Assoc 2006;137(Suppl):26S-31S

31. Grossi SG, Zambon JJ, Ho AW, Koch G, Dunford RG, Machtei EE, Norderyd OM, Genco RJ. Assessment of risk for periodontal disease. I. Risk indicators for attachment loss. J Periodontol 1994;65:260-7.

32. Morozumi T, Kubota T, Sato T, Okuda K, Yoshie H. Smoking cessation increases gingival blood flow and gingival crevicular fluid. J Clin Periodontol 2004;31(4):267-72. 
33. Löe $H$, Theilade $E$, Jensen SB. Experimental gingivitis in man. J Periodontol 1965;36:177-87.

34. Albandar JM. Global risk factors and risk indicators for periodontal diseases. Periodontol 2000 2002;29:177-206.

35. Mariotti A. Dental plaque- induced gingival diseases. Ann Periodontol 1999;4(1):7-17. 